\title{
PERFIL AUDIOMÉTRICO DE INDIVÍDUOS EXPOSTOS AO RUÍDO ATENDIDOS NO NÚCLEO DE SAÚDE OCUPACIONAL DE UM HOSPITAL DO MUNICÍPIO DE MONTES CLAROS, MINAS GERAIS
}

\author{
Audiometric profile of individuals exposed to noise attended \\ at the center for occupational health for a hospital in the municipality \\ of Montes Claros, Minas Gerais
}

\author{
Rejane Noronha Leão (1), Fernanda Abalen Martins Dias ${ }^{(2)}$
}

\begin{abstract}
RESUMO
Objetivo: traçar o perfil audiométrico de pacientes expostos a ruído, atendidos no Núcleo de Saúde Ocupacional da Fundação Hospitalar de Montes Claros (Hospital Aroldo Tourinho) em Minas Gerais. Métodos: análise de prontuários audiológicos do Núcleo de Saúde Ocupacional da Fundação Hospitalar de Montes Claros/MG de trabalhadores expostos a ruído ocupacional no período de junho a dezembro de 2006. Resultados: a amostra de prontuários caracterizou-se por predominância do sexo masculino (98,3\%), com média de 34,7 anos idade. Dos dados obtidos: $37,9 \%$ dos trabalhadores não usavam nenhum tipo de protetor auditivo; $19,8 \%$ dos trabalhadores foram expostos a um ou mais agente químico; $17 \%$ descreveram alguma queixa auditiva; $6,3 \%$ usavam algum medicamento; $7,4 \%$ citaram doenças prévias; $6,7 \%$ relataram deficientes auditivos na família. Identificou-se que $13,2 \%$ dos trabalhadores apresentaram perda auditiva na orelha direita $(31,8 \%$ destas perdas auditivas foram sugestivas de perda auditiva induzida por níveis elevados de pressão sonora) e $15,5 \%$ na orelha esquerda $(36,77 \%$ destas perdas auditivas foram sugestivas de perda auditiva induzida por níveis elevados de pressão sonora). Conclusão: nesta pesquisa houve uma alta prevalência de alterações audiométricas sugestivas de perda auditiva induzida por níveis elevados de pressão sonora, com predomínio maior de perdas auditivas na orelha esquerda. Também houve diferenças significantes em relação à exposição diária dos trabalhadores ao ruído, ao ruído extra-ocupacional e às funções desempenhadas pelos trabalhadores. Além disso, é preocupante o uso insuficiente e inadequado do equipamento de proteção individual auricular por parte dos trabalhadores expostos ao ruído.
\end{abstract}

DESCRITORES: Ruído; Ruído Ocupacional; Perda Auditiva; Audição; Perda Auditiva Provocada por Ruído

\section{INTRODUÇÃO}

A exposição a níveis de pressão sonora deletérios constitui-se em um importante agravo à saúde auditiva dos trabalhadores em todo o mundo. $\mathrm{O}$

\footnotetext{
(1) Fonoaudióloga Clínica, Montes Claros, MG.

(2) Fonoaudióloga; Docente do Curso de Especialização em Audiologia do CEFAC - Pós-graduação em Saúde e Educação, Belo Horizonte, MG; Mestre em Fonoaudiologia pela Pontifícia Universidade Católica de São Paulo.

Conflito de interesses: inexistente
}

agente físico nocivo mais comum encontrado no ambiente de trabalho é o ruído.

O ruído é um tipo de som que provoca uma sensação auditiva desagradável e que pode interferir na percepção de outros sons, principalmente os da fala. Os efeitos nocivos no ser humano dependem da intensidade e duração da exposição ao ruído, sendo que a exposição ao mesmo em excesso pode lesar considerável extensão das vias auditivas, desde a membrana timpânica até regiões do sistema nervoso central ${ }^{1}$. Os sintomas auditivos geralmente apresentados por indivíduos expostos 
ao ruído são: perda auditiva, zumbido, dificuldades na compreensão da fala ${ }^{1,2}$. Mas, também podem ocorrer sintomas extra-auditivos como alterações do sono e transtornos: da comunicação ${ }^{3}$, neurológicos, vestibulares, digestivos, comportamentais, cardiovasculares e hormonais ${ }^{1,2}$.

Os níveis de pressão sonora elevados impõem aos trabalhadores dificuldades de comunicação (na detecção, discriminação, localização e identificação da fonte sonora, assim como na inteligibilidade de fala) ${ }^{4}$, de manutenção da atenção e concentração, de memória, estresse ${ }^{1,2}$ e fadiga excessiva ${ }^{1}$. Estes são fatores associados a altas taxas de acidentes no trabalho ${ }^{1,4-6}$.

A exposição ao ruído é a principal causa de perda auditiva sensório-neural em indivíduos adultos. Atualmente, a Portaria 19/98 do Ministério do Trabalho e Emprego ${ }^{7}$ utiliza o termo perda auditiva induzida por níveis elevados de pressão sonora (PAINPSE) para definir a perda auditiva decorrente da exposição ocupacional contínua a intensos níveis de ruído. A PAINPSE é uma doença insidiosa, que acarreta perda auditiva progressiva e irreversível ${ }^{1,8}$, apresentando relação direta com a intensidade, tempo de exposição ${ }^{1,9}$ e a susceptibilidade individual do trabalhador ao ruído, entretanto, é passível de prevenção ${ }^{1}$. Caracteriza-se por diminuição gradual da acuidade auditiva num período de, geralmente, seis a dez anos de exposição a elevados níveis de pressão sonora, por qualquer exposição a uma intensidade a partir de $85 / 90 \mathrm{~dB}$ oito horas por dia. É quase sempre bilateral e simétrica, não ultrapassando $40 \mathrm{~dB}$ (NA) nas frequências graves e $75 \mathrm{~dB}$ (NA) nas frequências agudas; manifesta-se, primeiramente, em $6000 \mathrm{~Hz}{ }^{1,10}, 4000 \mathrm{~Hz}$ e/ou $3000 \mathrm{~Hz}$, estendendo-se às frequências de $8000 \mathrm{~Hz}, 2000 \mathrm{~Hz}$, $1000 \mathrm{~Hz}, 500 \mathrm{~Hz}$ e $250 \mathrm{~Hz}$ com a sua progressão ${ }^{1}$.

Uma pesquisa ${ }^{11} \mathrm{com}$ trabalhadores expostos a ruído realizada no Brasil demonstrou ser a exposição ao ruído e, consequentemente, a perda auditiva o problema de saúde ocupacional mais prevalente nos ambientes industriais, acometendo a audição de muitos trabalhadores.

A PAINPSE é uma das formas mais comuns de perda auditiva neurossensorial. Por isso, a necessidade de implantação de Programas de Conservação Auditiva (PCA) abrangendo trabalhadores expostos ao ruído ocupacional, para a intervenção na evolução das perdas auditivas geradas pela exposição ao ruído visando à manutenção da saúde auditiva, à diminuição dos sintomas associados ${ }^{8}$, ao controle da emissão de ruídos na fonte ${ }^{6}$, à diminuição considerável do risco de acidentes ${ }^{4,5}$.

Desta forma, o objetivo desse estudo é traçar o perfil audiométrico de pacientes expostos ao ruído ocupacional, atendidos no Núcleo de Saúde Ocu- pacional da Fundação Hospitalar de Montes Claros (Hospital Aroldo Tourinho) em Minas Gerais.

\section{MÉTODOS}

Após a autorização do Gerente de Ensino Pesquisa e Extensão e Gerente Administrativo Interino do Núcleo de Saúde Ocupacional da Fundação Hospitalar de Montes Claros (Hospital Aroldo Tourinho - HAT), foram analisados 1030 prontuários. Porém, fizeram parte da amostra somente 1003 prontuários de trabalhadores expostos a ruído ocupacional, nos quais constava repouso acústico de, pelo menos, 14 horas que antecedem ao exame.

O Núcleo de Saúde Ocupacional do HAT recebeu trabalhadores de várias empresas da cidade de Montes Claros para realização de exames audiométricos ocupacionais no período de junho a dezembro de 2006. Todos eles foram submetidos aos mesmos procedimentos: a) inspeção do meato acústico externo (MAE) a fim de descartar presença de corpos estranhos ou a existência de excesso de cerume, os quais pudessem comprometer a realização dos testes propostos; b) anamnese clínica ocupacional para investigar possíveis ocorrências passadas e/ou presentes de: otalgia, prurido, plenitude auricular, dificuldade em ouvir e/ou entender as pessoas falando, sensibilidade a sons de forte intensidade, presença de zumbido ou tontura, antecedente hereditário, diabetes, hipertensão arterial, se havia exposição ao ruído fora do ambiente de trabalho e/ou de outro tipo de agente responsável por alterações auditivas como: produtos químicos e uso de medicamentos ototóxicos; c) pesquisa dos limiares de audibilidade por via aérea nas frequências de $250 \mathrm{~Hz}$ a $8 \mathrm{kHz}$ e quando o limiar foi igual ou maior que $25 \mathrm{~dB}$, também foi realizada audiometria por via óssea nas frequências de $500 \mathrm{~Hz}$ a $4 \mathrm{kHz}$. O procedimento para a testagem por via aérea e por via óssea foi idêntico, utilizando-se de audiômetro calibrado anualmente de acordo com a Portaria 19 do Ministério do Trabalho.

Para o estudo foram analisadas as seguintes variáveis: sexo, idade, tipo de exames (admissional, periódico, retorno às atividades, mudança de função, demissional), tempo de atividade na empresa, tempo de exposição ao ruído ocupacional (estimado a partir do relato de cada trabalhador sobre a exposição ao ruído durante toda a sua vida laboral), tipo de ruído (constante, flutuante, impacto), uso regular de Equipamento de Proteção Individual Auricular (EPIA) (caracterizado pelo relato de utilização de protetor auricular por parte dos trabalhadores sempre que exerciam atividades em ambientes barulhentos), exposição extra-ocupacional ao ruído (hábito de uso de armas de fogo, ou de 
frequentar festas barulhentas, ou de soltar foguetes e/ou bombas), exposição ocupacional a produtos químicos (ácidos, solventes, redutores, óleos e graxas), história familiar de déficit auditivo, doenças atuais e pregressas (hipertensão arterial, diabetes mellitus), queixas de origem otológica (hipoacusia, tinnitus, vertigem), uso de medicamento ototóxico e achados audiológicos. Foram adotados como critérios de exclusão os pacientes que apresentavam repouso acústico inferior a 14 horas que antecedem ao exame bem como aqueles que estavam expostos a ruído inferior a $85 \mathrm{~dB}$ NPS.

Os achados audiológicos foram classificados segundo critérios de normalidade - quando os limiares auditivos por via aérea forem menor ou igual a $25 \mathrm{~dB}$ - e diagnósticos de diferenciação entre perdas dos tipos: neurossensorial - quando os limiares auditivos de via aérea e via óssea estão rebaixados, sem presença de gap; condutiva - quando via aérea encontra-se com limiares auditivos rebaixados e a via óssea com limiares auditivos preservados, com presença de gap maior ou igual a $10 \mathrm{~dB}$; mista - quando existe um componente condutivo associado a um componente neurossensorial, ou seja, há um rebaixamento auditivo nos limiares de via aérea e via óssea, porém com um gap entre eles maior que 10dB; sugestiva de PAINPSE quando as frequências de 3.000 e/ou 4.000 e/ou $6.000 \mathrm{~Hz}$ apresentarem limiares auditivos acima de $25 \mathrm{~dB}(\mathrm{NA})$ e mais elevados do que nas outras frequências testadas, estando estas comprometidas ou não, tanto no teste da via aérea quanto da via óssea, em um ou em ambos os lados; e, não sugestiva de PAINPSE - quando as alterações no audiograma não se enquadram nas descrições acima, ou seja, frequências isoladas, que não sejam as de 3.000 e/ou 4.000 e/ou $6.000 \mathrm{~Hz}$, apresentarem limiares auditivos acima de $25 \mathrm{~dB}$ (NA) e mais elevados do que nas outras frequências testadas, estando estas comprometidas ou não, tanto no teste da via aérea quanto da via óssea, em um ou em ambos os lados ${ }^{7}$.

Este estudo foi aprovado pelo Comitê de Ética em Pesquisa do CEFAC - Saúde e Educação, sob o número 117/07, datado em 12/09/07 e foi considerado sem risco, mas com necessidade do Termo de Consentimento Livre e Esclarecido ou de Compromisso de, ao utilizar dados e/ou informações coletados no(s) prontuários do(s) sujeito(s) da pesquisa, assegurar a confidencialidade e a privacidade dos mesmos.

Para a análise estatística foi utilizado 0 software Epilnfo 2000, versão 3.3.2. Foram calculadas médias e percentuais das variáveis e feita análise descritiva. Foi utilizado quando necessário, o teste do Qui-quadrado - Yates $\left(X^{2}{ }_{\text {(Yates) }}\right)$ na aferição das diferenças observadas entre os diversos grupos comparados neste estudo. Todas as conclusões foram obtidas para um nível de significância de no máximo $5 \%$ ( $p<0,05)$, ou seja, com pelo menos $95 \%$ de confiança nos resultados.

\section{RESULTADOS}

A amostra de 1003 prontuários de trabalhadores analisados caracterizou-se por predominância quase absoluta de 986 do sexo masculino $(98,3 \%)$, com média de 34,7 anos (idade mínima de 18 anos e máxima de 74 anos). Os trabalhadores da amostra exerciam vários tipos de funções, estas foram agrupadas e apresentadas na Figura 1 da seguinte forma: não souberam referir a função a qual exerciam $(12,8 \%)$, serviços gerais $(24,2 \%)$, ajudante de produção $(11,5 \%)$, motorista $(24,0 \%)$, vendedor $(1,8 \%)$, mecânico/auxiliar de mecânico $(14,7 \%)$, assistente administrativo $(3,8 \%)$, pedreiro/auxiliar de pedreiro $(4,8 \%)$ e instrutor/coordenador $(2,5 \%)$. $\mathrm{Na}$ Figura 2, encontra-se a distribuição do ramo de atuação das empresas em que os trabalhadores desempenham suas funções laborais: $2,5 \%$ saneamento; $0,3 \%$ hospitalar; $1,4 \%$ escola técnica; $11 \%$ agrícola; $21,5 \%$ pavimentação; $11,6 \%$ indústria; 5,9\% construção; $1,2 \%$ mineração; $23,8 \%$ fábrica; $11 \%$ transporte; $9,5 \%$ oficina; e $0,4 \%$ gráfica.

O presente estudo foi feito a partir de uma investigação restrita a um dado período, não sendo possível comparar as audiometrias mais recentes com as anteriores (audiometria de referência), impossibilitando, assim, o monitoramento audiológico dos trabalhadores. Entretanto, pode se verificar que 382 exames $(38,1 \%$ da amostragem) foram de exames admissionais; 465 exames (46,4\% da amostragem) de periódicos; dois exames $(0,2 \%$ da amostragem) de retorno às atividades; um exame $(0,1 \%$ da amostragem) de mudança de função; e 153 exames (15,3\% da amostragem) demissionais.

Em relação ao tempo total de atividades em ambiente ruidoso, $36,9 \%$ dos trabalhadores relataram menos que 5 anos; $17,4 \%$ de 5 a 10 anos; $22,8 \%$ de 10 a 20 anos; $10,3 \%$ de 20 a 30 anos; $1,9 \%$ mais de 30 anos; e 10,7\% ainda não exerciam ou naquele momento não estavam desempenhando atividades em ambiente ruidoso. Aproximadamente $57 \%$ dos trabalhadores afirmaram estarem expostos a ruídos de 8 a 10 horas por dia.

As Tabelas 1 e 2 mostram comparações entre os tempos de atividades dos trabalhadores em ambiente ruidoso na empresa atual e em empresas anteriores à atual bem como a atual exposição diária e exposições diárias anteriores à atual. Nestas comparações as diferenças foram significantes apresentando uma probabilidade de 0,0002 para os tempos de atividades em ambiente ruidoso e de 0,0000 para exposições diárias ( $p$ menor que 0,05 ). 


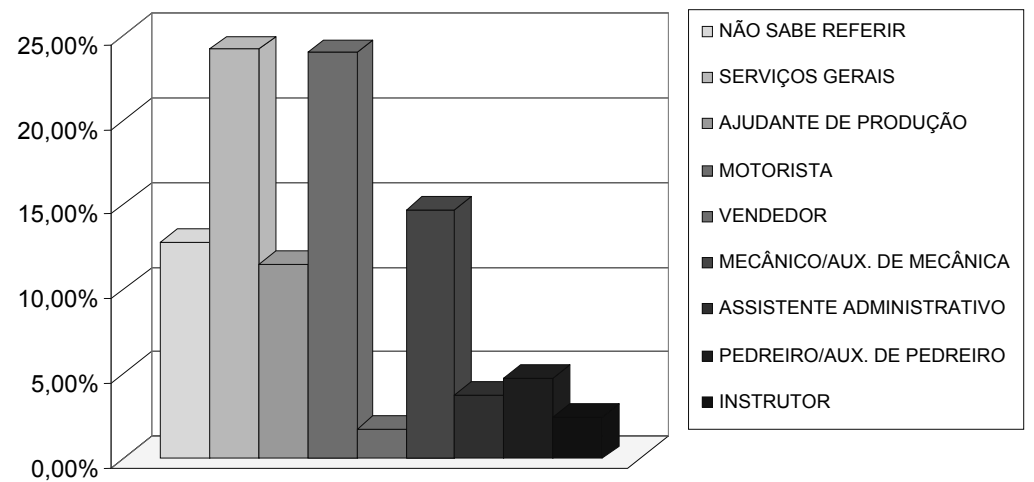

Figura 1 - Funções desempenhadas pelos trabalhadores

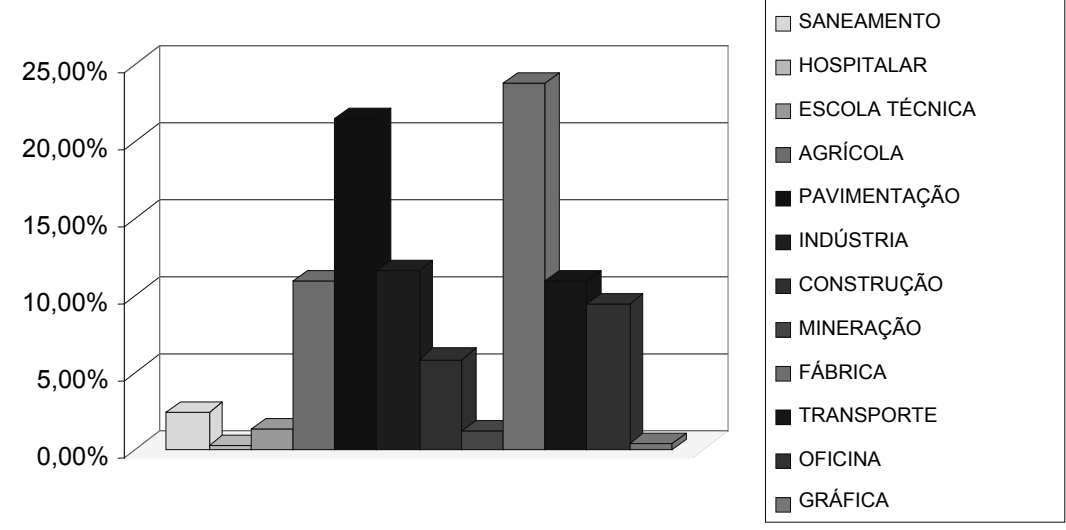

Figura 2 - Ramo de atuação das empresas em que os trabalhadores desempenham suas funções laborais

Tabela 1 - Tempo de atividade dos trabalhadores em ambiente ruidoso

\begin{tabular}{lccc}
\hline & ANTERIOR & ATUAL & TOTAL \\
\hline < 5 ANOS & $35,1 \%$ & $36,4 \%$ & $36,9 \%$ \\
5 A 10 ANOS & $12,9 \%$ & $12,4 \%$ & $17,4 \%$ \\
10 A 20 ANOS & $12,9 \%$ & $10,5 \%$ & $22,8 \%$ \\
20 A 30 ANOS & $3,7 \%$ & $3,6 \%$ & $10,3 \%$ \\
> 30 ANOS & $0,6 \%$ & $0,5 \%$ & $1,9 \%$ \\
NÃO TRABALHA EM RUÍDO & $34,9 \%$ & $36,7 \%$ & $10,7 \%$ \\
TOTAL & $100 \%$ & $100 \%$ & $100 \%$ \\
\hline
\end{tabular}

Teste Qui-Quadrado: $p=0.0002$ (significante)

Tabela 2 - Exposição diária dos trabalhadores em ambiente ruidoso

\begin{tabular}{lcc}
\hline & ANTERIOR & ATUAL \\
\hline$<8$ HORAS & $0,9 \%$ & $0,3 \%$ \\
$8-10$ HORAS & $57,8 \%$ & $57,6 \%$ \\
$10-12$ HORAS & $6,4 \%$ & $5,4 \%$ \\
$>12$ HORAS & - & $0,2 \%$ \\
NÃO TRABALHA EM RUÍDO & $34,9 \%$ & $36,5 \%$ \\
TOTAL & $100 \%$ & $100 \%$ \\
\hline
\end{tabular}

Teste Qui-Quadrado: $p=0.0000$ (significante) 
Quanto aos tipos de ruído, na atual empresa $32,9 \%$ dos trabalhadores referiram exposição ao ruído constante, $20,3 \%$ ao ruído flutuante ou intermitente, $5,6 \%$ ao ruído de impacto e $41,2 \%$ não souberam referir o tipo de ruído a que foram expostos (Tabela 3).

Na comparação do ruído na atual empresa com o ruído nas empresas anteriores, a diferença foi significante $p=0,0000(p<0,05)$. Dos trabalhadores participantes, $12,3 \%$ também referiram exposição ao ruído extra-ocupacional.

No tocante ao uso de Equipamento de Proteção Individual Auditivo (EPIA) na atual empresa, dos $63,1 \%$ dos trabalhadores que estavam expostos ao ruído, $62,1 \%$ deles usavam algum tipo de protetor auricular (plugue ou abafador, ou até mesmo, os dois juntos), mas 37,9\% não usavam nenhum tipo de protetor auricular (Tabela 4). Houve uma diferença significante na comparação do uso de EPIA feita na empresa atual e nas anteriores à atual $p=$ $0,0000(p<0,05)$.

Foi verificado, que $19,8 \%$ dos trabalhadores foram expostos a um ou mais agentes químicos, $17 \%$ descreveram alguma queixa auditiva (destas, $11,17 \%$ foram relativas a zumbido), $6,3 \%$ usavam algum medicamento $(0,1 \%$ para diabetes, $3,9 \%$ para hipertensão arterial, $0,6 \%$ anti-inflamatórios e 1,7\% outros), $7,4 \%$ citaram doenças prévias $(1,6 \%$ otites, $0,1 \%$ diabetes, $4 \%$ hiper- tensão arterial, $0,2 \%$ otite e hipertensão arterial e $1,5 \%$ outras), $6,7 \%$ relataram deficientes auditivos na família (destes $0,7 \%$ são os próprios trabalhadores).

$\mathrm{Na}$ análise dos achados audiométricos, identificou-se que $13,2 \%$ dos trabalhadores apresentaram perda auditiva na orelha direita, sendo $0,1 \%$ anacusia, $0,3 \%$ condutiva, $0,7 \%$ mista, $2,2 \%$ neurossensorial, 5,7\% não sugestiva de PAINPSE e 4,2\% sugestiva de PAINPSE $(31,8 \%$ das perdas auditivas), ainda: $15,5 \%$ apresentaram perda auditiva na orelha esquerda, sendo $0,1 \%$ anacusia, $0,8 \%$ condutiva, $0,3 \%$ mista, $2,1 \%$ neurossensorial, $6,5 \%$ não sugestiva de PAINPSE e 5,7\% sugestiva de PAINPSE $(36,77 \%$ das perdas auditivas). Com isso, foi verificado predomínio de perda auditiva unilateral à esquerda (Figura 3). Houve uma diferença significante quando feita a comparação entre as orelhas $p=0,0000(p<0,05)$. Também houve diferença significante quando relacionadas às oreIhas com a exposição diária dos trabalhadores ao ruído (orelha direita: $p=0,0012(p<0,05)$ e orelha esquerda: $p=0,0003(p<0,05))$, com o ruído extraocupacional (orelha direita: $p=0,0175(p<0,05)$ e orelha esquerda: $p=0,0000(p<0,05))$ e com as funções desempenhadas pelos trabalhadores (oreIha esquerda: $p=0,0007(p<0,05))$. Nesta comparação somente a relação com a orelha esquerda foi significante.

Tabela 3 - Tipos de ruído relatados pelos trabalhadores

\begin{tabular}{lcc}
\hline & ANTERIOR & ATUAL \\
\hline CONSTANTE & $35,8 \%$ & $32,9 \%$ \\
FLUTUANTE & $18,9 \%$ & $20,3 \%$ \\
IMPACTO & $5,0 \%$ & $5,6 \%$ \\
NÃO TRABALHA EM RUÍDO & $40,3 \%$ & $41,2 \%$ \\
TOTAL & $100 \%$ & $100 \%$ \\
\hline
\end{tabular}

Teste Qui-Quadrado: $p=0.0000$ (significante)

Tabela 4 - Equipamento de proteção individual auditivo utilizado pelos trabalhadores

\begin{tabular}{lcc}
\hline & ANTERIOR & ATUAL \\
\hline ABAFADOR & $12,0 \%$ & $10,8 \%$ \\
PLUG & $23,2 \%$ & $26,4 \%$ \\
PLUG + ABAFADOR & $5,0 \%$ & $2,0 \%$ \\
NÃO USA & $24,3 \%$ & $23,9 \%$ \\
NÃO TRABALHA EM RUÍDO & $35,5 \%$ & $36,9 \%$ \\
TOTAL & $100 \%$ & $100 \%$ \\
\hline
\end{tabular}

Teste Qui-Quadrado: $p=0.0000$ (significante) 


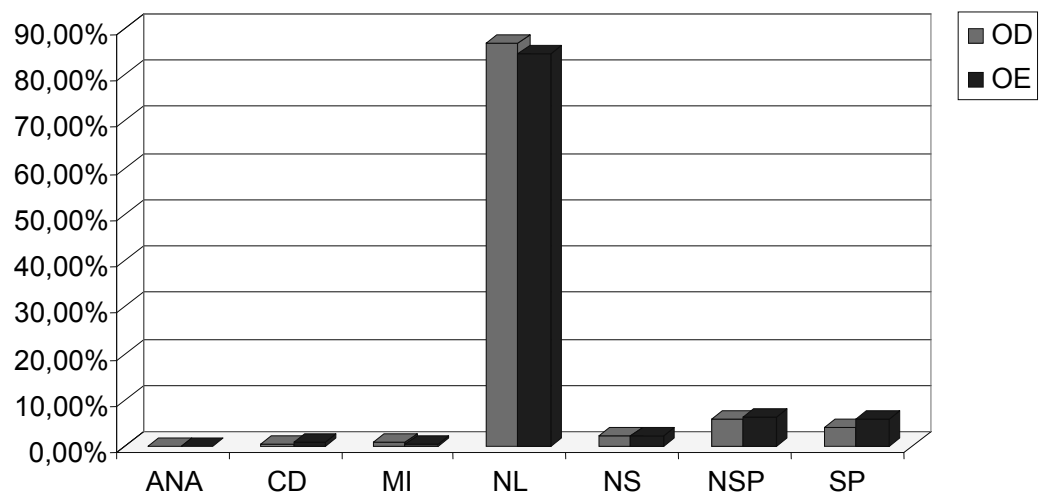

LEGENDA: ANA - ANACUSIA; CD - CONDUTIVA; MI - MISTA; NL - NORMAL; NS - NEUROSSENSORIAL; NSP - NÃO SUGESTIVA DE PAINPSE; SP - SUGESTIVA DE PAINPSE

Teste Qui-Quadrado: $p=0.0000$ (significante)

\section{Figura 3 - Resultados audiométricos dos exames realizados nos trabalhadores}

\section{DISCUSSÃO}

As alterações auditivas encontradas neste estudo se assemelham com a pesquisa ${ }^{11}$ que encontrou $19,6 \%$ para quaisquer alterações auditivas; mas não se confirmam com outros autores ${ }^{11}$, pois os valores encontrados por estes foram muito superiores aos achados nesta pesquisa. Em muitos estudos ${ }^{2,11}$ ocorrem também associação entre perdas auditivas e agentes químicos utilizados pelos trabalhadores expostos, o que torna importante ser mencionada a prevalência de agentes químicos neste estudo que foi de $19,8 \%$.

Autores ${ }^{12}$ que avaliaram trabalhadores expostos a ruídos intensos em São Paulo, encontraram prevalências de alterações auditivas entre 30 e $55 \%$, de acordo com o ramo de atividade. Também avaliando trabalhadores de diversos setores industriais em Salvador, pesquisadores ${ }^{10}$ encontraram prevalência geral de PAINPSE em torno de $36 \%$. Dentre as alterações auditivas encontradas nessa casuística, a prevalência de casos sugestivos de PAINPSE foi de $31,8 \%$ para a orelha direita e de $36,77 \%$ para a orelha esquerda, que corrobora, de certa forma, a variação de 28,5 a $46,2 \%$ encontradas em estudo anterior ${ }^{10} \mathrm{e}$ se aproximam também dos resultados obtidos em outra pesquisa ${ }^{11}$, em torno de 30 a $36 \%$ de perda auditiva sugestiva de PAINPSE unilateral. Isso confirma também o predomínio de perda auditiva unilateral à esquerda ${ }^{10,11,13}$.

$O$ uso regular de EPIA em relação a todos os analisados nesta pesquisa foi de $39,2 \%$ que se aproximaram do relato de alguns autores ${ }^{13}$. Entretanto, em se tratando somente dos trabalhadores que referiram exposição a ruído a porcentagem foi de $62,1 \%$, que está bem abaixo de outro estudo realizado ${ }^{1}$. Isso mostra a necessidade de se enfatizar a indicação, adequação e acompanhamento do uso do EPIA, mesmo quando as medidas de proteção coletiva forem insatisfatórias.

Pesquisadores ${ }^{9}$ referem uma associação significante entre perda auditiva induzida por ruído e nível de utilização de protetores auriculares. Alguns estudiosos ${ }^{4}$ citaram que trabalhadores sem proteção auditiva atuando em ambientes ruidosos têm maior chance de acidentarem-se.

A exposição ao ruído também é responsável pela causa mais comum de zumbido, referido por cerca de $25 \%$ dos indivíduos expostos ${ }^{14}$. $\mathrm{Na}$ casuística do presente estudo, dentre os sintomas auditivos referidos pelos indivíduos expostos, a prevalência de zumbido encontrada ficou em torno de $11,17 \%$, muito inferior àquela referida pela literatura ${ }^{9}(62,5 \%)$; entretanto, aproximou-se ao valor encontrado em outros estudos ${ }^{1,13}$.

Zumbidos e perdas auditivas são os sintomas mais comuns de serem relatados após exposição ao ruído intenso causador do trauma acústico. Neste sentido, foi pesquisada a exposição extraocupacional, sendo que $12,3 \%$ dos sujeitos expostos ao ruído relataram frequentar danceterias, shows, fazerem serviços com equipamentos baruIhentos sem uso de nenhum tipo de proteção auditiva ou foram expostos a algum tipo de explosão (arma de fogo ou fogos de artifício). Estes dados estão de acordo com um estudo ${ }^{1}$ em relação a trauma acústico (12\%). Outros autores ${ }^{9}$ também identificaram prevalências de casos sugestivos de PAINPSE maiores nos trabalhadores com história de exposição a fontes de ruído extra-ocupacional (25,0\%).

Também é notável a produção de trabalhos sobre associações entre a perda auditiva induzida por níveis elevados de pressão sonora e fatores 
como hipertensão arterial sistêmica e diabetes mellitus. Neste estudo, a prevalência desses fatores foi de 4,2\% hipertensão arterial, 0,1\% diabetes. Estes dados se mostraram abaixo dos relatados pela literatura ${ }^{9}$ (hipertensão arterial $22,7 \%$, diabetes mellitus 30,0\%).

E ainda, não menos importante, $6,8 \%$ dos trabalhadores informou história de déficit auditivo na família. Nos estudos de alguns pesquisadores ${ }^{9} \mathrm{a}$ prevalência de casos sugestivos de PAINPSE foi menor entre os trabalhadores com história familiar de déficit auditivo em relação aos trabalhadores sem o relato dessas histórias.

As ações consideradas como sendo de preservação da audição dos trabalhadores nas empresas atendidas pelo Núcleo de Saúde Ocupacional do HAT são predominantemente baseadas na reali- zação de audiometrias ocupacionais e no fornecimento de protetores auriculares por algumas dessas empresas, caso as mesmas acreditem serem necessários.

Assim, em face dos resultados, torna-se fundamental e justificável o acompanhamento desses pacientes, objetivando a manutenção da saúde auditiva dos mesmos.

\section{CONCLUSÃO}

Houve uma alta prevalência de perda auditiva sugestiva de PAINPSE, com predomínio maior de perdas auditivas na orelha esquerda. Além disso, foi constatado uso insuficiente do EPIA por parte dos trabalhadores expostos ao ruído e os que fazem uso, muitas vezes, o fazem inadequadamente.

\begin{abstract}
Purpose: to draw the audiometric profile of patients exposed to noise, attended the Center for Occupational Health Foundation's Hospital of Montes Claros (Aroldo Tourinho Hospital) in Minas Gerais. Methods: analysis of audiological records of the Center for Occupational Health of Foundation Hospital of Montes Claros / MG of workers exposed to occupational noise in the period June to December 2006. Results: the sample of medical records characterized by male predominance $(98.3 \%)$, with a mean age of 34.7 years. Data obtained as follows: $37.9 \%$ of workers were not using any type of hearing protector, $19.8 \%$ of workers were exposed to one or more chemical agent, $17 \%$ reported hearing any complaints, $6.3 \%$ used some medicine, $7,4 \%$ cited previous diseases, $6.7 \%$ reported hearing disability in the family. It was identified that $13.2 \%$ of workers had hearing loss in right ear $(31.8 \%$ of hearing loss were suggestive of hearing loss induced by high levels of sound pressure) and $15.5 \%$ in the left ear (36.77\% of hearing loss were suggestive of hearing loss induced by high levels of sound pressure). Conclusion: in this study there was a high prevalence of suggestive audiometric alterations concerning hearing loss induced by high levels of sound pressure, with higher prevalence of hearing loss in the left ear. There were also significant differences in relation to workers' daily exposure to noise, noise and non-occupational tasks performed by the workers. Moreover, the inadequate and inappropriate use of personal auricular protective equipment by workers exposed to noise is preoccupying.
\end{abstract}

KEYWORDS: Noise; Noise, Occupational; Hearing Loss; Hearing; Hearing Loss, Noise-Induced

\section{REFERÊNCIAS}

1. Araújo SA. Perda auditiva induzida pelo ruído em trabalhadores de metalúrgica. Rev Bras Otorrinolaringol. 2002; 68(1):47-52.

2. Gonçalves CGO, Iguti AM. Análise de programas de preservação da audição em quatro indústrias metalúrgicas de Piracicaba, São Paulo, Brasil. Cad Saúde Pública. 2006; 22(3):609-18.

3. Marques FP, Costa EA. Exposição ao ruído ocupacional: alterações no exame de emissões otoacústicas. Rev Bras Otorrinolaringol. 2006; 72(3):362-6.

4. Berger EH, Royster LH, Royster JD, Driscoll DP, Layne M. The noise manual. 5. ed. Akron: American Industrial Hygiene Association; 2000.

5. Dias A, Cordeiro R, Gonçalves CGO. Exposição ocupacional ao ruído e acidentes do trabalho. Cad Saúde Pública. 2006; 22(10):2125-30.

6. Cordeiro R, Clemente APG, Diniz CS, Dias A. Exposição ao ruído ocupacional como fator de risco para acidentes do trabalho. Rev Saúde Pública. 2005; 39(3): 461-6. 
7. Brasil. Ministério do Trabalho. Secretaria de Segurança do Trabalho. Portaria n.19 de 9 de abril de 1998. Brasília (DF): Diário Oficial da União; 1998. 8. Dias A, Cordeiro R, Corrente JE, Gonçalves CGO. Associação entre perda auditiva induzida pelo ruído e zumbidos. Cad Saúde Pública. 2006; 22(1):63-8.

9. Guerra MR, Lourenço PMC, Bustamante-Teixeira MT, Alves MJM. Prevalência de perda auditiva induzida por ruído em empresa metalúrgica. Rev Saúde Pública. 2005; 39(2): 238-44.

10. Miranda CR, Dias CR, Pena PGL, Nobre LCC, Aquino R. Perda auditiva induzida pelo ruído em trabalhadores industriais da região metropolitana de Salvador, Bahia. IESUS. 1998; 7(1):87-94.

11. Franco ES. Prevalência de perdas auditivas em trabalhadores no processo de admissão em empresas da região de Campinas SP [dissertação]. São Paulo (SP): Pontifícia Universidade Católica de São Paulo; 2000.

12. Andrade CRF, Schochat E. Perfil audiométrico de trabalhadores de indústrias ruidosas. In: Anais do I Encontro Nacional de Fonoaudiologia Social e Preventiva. São Paulo: Conselho Regional de Fonoaudiologia 2a Região; 1988. p. 71-81.

13. Silva AP, Costa EA da, Rodrigues SMM, Souza HLR, Massafera VG. Avaliação do perfil auditivo de militares de um quartel do Exército Brasileiro. Rev Bras Otorrinolaringol. 2004; 70(3):344-50.

14. Sahley TL, Nodar RH. Tinnitus: present and future. Curr Opin Otolaryngol Head Neck Surg. 2001; 9(5):323-8.

RECEBIDO EM: 24/03/2009

ACEITO EM: 17/10/2009

Endereço para correspondência:

Rejane Noronha Leão

Rua General Carneiro, 553

Montes Claros - MG

CEP: 39400-095

E-mail: reje.leao@yahoo.com.br 